\title{
HOMOGENEOUS SYMMETRIC ANTIASSOCIATIVE QUASIALGEBRAS
}

\author{
HELENA ALBUQUERQUE, ELISABETE BARREIRO, AND SAÏD BENAYADI
}

\begin{abstract}
Our main purpose is to provide for homogeneous (even or odd) symmetric antiassociative quasialgebras a structure theory analogous to that for homogeneous symmetric associative superalgebras given in [5] and to present an inductive description of these classes of algebras.
\end{abstract}

\section{INTRODUCTION}

The $\mathbb{Z}_{2}$-graded quasialgebras are formed by two classes of algebras: the associative superalgebras well known from the work of C.T.C. Wall in 1964 and M.L. Racine in 1998 (see [8] and [7]); and the class of antiassociative quasialgebras introduced by H. Albuquerque and S. Majid in 1999, and later addressed by H. Albuquerque, A. Elduque and J.M. Pérez-Izquierdo in 2002 (see [2] and [1]). These two classes of algebras have non-empty intersection, for example, associative algebras belong to both classes.

Recently, it was studied the class of homogeneous symmetric associative superalgebras by I. Ayadi and S. Benayadi (see $[4,5,6]$ ). The authors presented an inductive description of homogeneous symmetric associative superalgebras using generalized double extension of associative superalgebras. They also gave a description of odd symmetric associative superalgebras whose even part is semisimple bimodule, without using double extension process. It appears the question of how can be performed the description of homogeneous symmetric antiassociative quasialgebras.

The aim of this paper is to respond to this question, completing the study of $\mathbb{Z}_{2}$-graded quasialgebras provided with homogeneous symmetric structures. The results obtained are closed related to those of homogeneous symmetric associative superalgebras, being the proofs similar in some details, but different in general.

The paper is structured as follows. First we give some definitions and previous results needed to describe the homogeneous symmetric antiassociative quasialgebras. In the second section we find out which of the simple antiassociative quasialgebras admit homogeneous structures. The next one is devoted to the odd-symmetric antiassociative quasialgebras and we present a description of this class of algebras. It is important to stress that the class of odd-symmetric antiassociative quasialgebras is contained in the class of associative superalgebras. The fourth section introduces the notion of generalized double extension of even-symmetric antiassociative quasialgebras. As a goal of the paper, last sections present a description of even-symmetric antiassociative quasialgebras with even part semi-simple bimodule, and a description of the even-symmetric antiassociative quasialgebras in general.

Throughout this paper, we work with finite dimensional algebras over an algebraically closed commutative field $\mathbb{K}$ of characteristic zero. We start by dealing with some definitions and previous results needed in the sequel.

The first and the second authors acknowledge partial financial assistance by CMUC and FCT (Portugal), through European program COMPETE/FEDER. 
Definition 1.1. [2] Let $G$ be a group, $\mathbb{K}$ a field and $\phi: G \times G \times G \longrightarrow \mathbb{K}^{\times}$ a invertible $G$-cocycle. A $G$-graded quasialgebra is a $G$-graded $\mathbb{K}$-algebra $A=$ $\bigoplus_{g \in G} A_{g}$ with product map $A \otimes A \longrightarrow A$ preserving the total degree and obeying to the quasiassociative law in the sense

$$
(x y) z=\phi(\bar{x}, \bar{y}, \bar{z}) x(y z), \quad \forall x \in A_{\bar{x}}, y \in A_{\bar{y}}, z \in A_{\bar{z}}
$$

being $\bar{x} \in G$ for arbitrary homogeneous elements $x \in A$. We will denote the quasialgebra by the pair $(A, \phi)$.

We recall that $\phi: G \times G \times G \longrightarrow \mathbb{K}^{\times}$is $G$-cocycle if

$$
\phi(y, z, w) \phi(x, y z, w) \phi(x, y, z)=\phi(x, y, z w) \phi(x y, z, w) \text { and } \phi(x, e, y)=1,
$$

holds for all $x, y, z, w$ in $G$, where $e$ denotes the identity of the group $G$.

Definition 1.2. Let $A=\bigoplus_{g \in G} A_{g}$ be a $G$-graded quasialgebra, $V=\bigoplus_{g \in G} V_{g}$ a $G$-graded vector space and $\varphi: A \times V \longrightarrow V, \psi: V \times A \longrightarrow V$ two bilinear maps denoted by $\varphi(x, v)=x . v$ and $\psi(v, x)=v . x$, with $x \in A, v \in V$. If for homogeneous elements $x \in A_{\bar{x}}, y \in A_{\bar{y}}, v \in V_{\bar{v}}$,

$$
\begin{aligned}
& (x y) \cdot v=\phi(\bar{x}, \bar{y}, \bar{v}) x \cdot(y \cdot v), \\
& (x \cdot v) \cdot y=\phi(\bar{x}, \bar{y}, \bar{v}) x \cdot(v \cdot y), \\
& (v \cdot x) \cdot y=\phi(\bar{x}, \bar{y}, \bar{v}) v \cdot(x y),
\end{aligned}
$$

then $V$ will be called a quasi-bimodule of $A$ (or a A-quasi-bimodule).

In this paper, we are interested in the simplest nontrivial group of two elements $G=\mathbb{Z}_{2}$ (assuming characteristic of $\mathbb{K}$ not 2 ). In this case only occur two possibilities for the cocycle $\phi$, either $\phi(\overline{1}, \overline{1}, \overline{1})=1$ or -1 and all the other values $\phi(\bar{x}, \bar{y}, \bar{z})$ being always 1 . Thus any $\mathbb{Z}_{2}$-graded quasialgebra $A=A_{\overline{0}} \oplus A_{\overline{1}}$ is either associative or it satisfies the "antiassociative" condition $(x y) z=(-1)^{\bar{x} \bar{y} \bar{z}} x(y z)$ for homogeneous elements $x, y, z \in A_{\overline{0}} \cup A_{\overline{1}}$.

Definition 1.3. [1] An antiassociative quasialgebra (aaq-algebra for short) is a $\mathbb{Z}_{2^{-}}$ graded quasialgebra $A=A_{\overline{0}} \oplus A_{\overline{1}}$ over an unital commutative and associative ring of scalars satisfying

$$
(x y) z=(-1)^{\bar{x} \bar{y} \bar{z}} x(y z), \quad \forall x, y, z \in A_{\overline{0}} \cup A_{\overline{1}} .
$$

From the definition we see that $A_{\overline{0}}$ is an associative algebra and $A_{\overline{1}}$ is a $A_{\overline{0}}$-quasibimodule with structure defined by: $\forall x \in A_{\overline{0}}, y \in A_{\overline{0}}, v \in A_{\overline{1}}$,

$$
(x y) \cdot v=x \cdot(y \cdot v), \quad(x \cdot v) \cdot y=x \cdot(v \cdot y), \quad(v \cdot x) \cdot y=v \cdot(x y) .
$$

If $A=A_{\overline{0}} \oplus A_{\overline{1}}$ is an antiassociative quasialgebra then there is an "antiassociative" multiplication $A_{\overline{1}} \otimes_{A_{\overline{0}}} A_{\overline{1}} \longrightarrow A_{\overline{0}}$ which is a homomorphism of $A_{\overline{0}}$-quasi-bimodules.

Proposition 1.4. The intersection of the class of associative superalgebras with the class of antiassociative quasialgebras is the set of the $\mathbb{Z}_{2}$-graded quasialgebras $A=A_{\overline{0}} \oplus A_{\overline{1}}$ satisfying $A_{\overline{1}}^{2} A_{\overline{1}}=\{0\}$.

Proof. If $A=A_{\overline{0}} \oplus A_{\overline{1}}$ is a $\mathbb{Z}_{2}$-graded quasialgebra such that $A_{\overline{1}}^{2} A_{\overline{1}}=\{0\}$ then obviously it belongs to the intersection considered in the proposition. Conversely, take $A=A_{\overline{0}} \oplus A_{\overline{1}}$ in the intersection of the class of associative superalgebras and of antiassociative quasialgebras. Let us assume that $A_{\overline{1}}^{2} A_{\overline{1}} \neq\{0\}$. So there exist $x, y, z \in A_{\overline{1}}$ such that $(x y) z \neq 0$. Since $A$ is an associative superalgebra then $(x y) z=x(y z)$ and as $A$ is an antiassociative quasialgebra we have $(x y) z=$ $-x(y z)$. As characteristic of $\mathbb{K}$ is not 2 , we conclude that $(x y) z=0$, which is a contradiction. 
Let $A=A_{\overline{0}} \oplus A_{\overline{1}}$ be a $\mathbb{Z}_{2}$-graded quasialgebra in the intersection referred in the previous result, meaning that $A_{\overline{1}}^{2} A_{\overline{1}}=\{0\}$. Then occurs three distinct possibilities:

(1) If $A_{\overline{1}}=\{0\}$, then $A$ is an associative algebra;

(2) If $A_{\overline{1}} \neq\{0\}$ and $A_{\overline{1}}^{2}=\{0\}$, so $A$ is a trivial $\mathbb{Z}_{2}$-graded quasialgebra;

(3) If $A_{\overline{1}}^{2} \neq\{0\}$, the most relevant case.

Definition 1.5. A division antiassociative quasialgebra (division aaq-algebra for short) is an antiassociative quasialgebra $A=A_{\overline{0}} \oplus A_{\overline{1}}$ such that it is unital $\left(1 \in A_{\overline{0}}\right)$ and every nonzero homogeneous element has a left and right inverses.

In a division antiassociative quasialgebra the left and right inverses coincide for an even element, but they differ in sign for an odd element. The following division aaq-algebra has an important role in the study of aaq-algebras [1] and it will appear later on. Let $D$ be a division (associative) algebra, $\sigma: D \longrightarrow D$ an automorphism of $D$ such that there exists an element $0 \neq a \in D$ with $\sigma^{2}=\tau_{a}: d \longrightarrow a d a^{-1}$ and $\sigma(a)=-a$. On the direct sum of two copies of $D: \Delta=D \oplus D u$ (here $u$ is just a marking device) define the following multiplication: for all $d_{i}, e_{i} \in D(i=0,1)$

$$
\left(d_{0}+d_{1} u\right)\left(e_{0}+e_{1} u\right)=\left(d_{0} e_{0}+d_{1} \sigma\left(e_{1}\right) a\right)+\left(d_{0} e_{1}+d_{1} \sigma\left(e_{0}\right)\right) u .
$$

Taking $\Delta_{\overline{0}}=D$ and $\Delta_{\overline{1}}=D u$, it is easy to show that $\Delta=\Delta_{\overline{0}} \oplus \Delta_{\overline{1}}$ is a division aaq-algebra and it is denoted by $\langle D, \sigma, a\rangle$.

Theorem 1.6. [1] Any division aaq-algebra with nonzero odd part is, up to an isomorphism, an aaq-algebra $\langle D, \sigma, a\rangle$.

Definition 1.7. Let $A$ be an $\mathbb{Z}_{2}$-graded quasialgebra and $B$ a bilinear form on $A$.

(i) $B$ is called supersymmetric if $B(x, y)=(-1)^{\bar{x} \bar{y}} B(y, x), \forall x, y \in A_{\overline{0}} \cup A_{\overline{1}}$;

(ii) $B$ is called non-degenerate if $x \in A$ satisfies $B(x, y)=0, \forall y \in A$, then $x=0$;

(iii) $B$ is called associative if $B(x y, z)=B(x, y z), \forall x, y, z \in A$;

(iv) $B$ is called even if $B\left(A_{\overline{0}}, A_{\overline{1}}\right)=\{0\}$;

(v) $B$ is called odd if $B\left(A_{\overline{0}}, A_{\overline{0}}\right)=B\left(A_{\overline{1}}, A_{\overline{1}}\right)=\{0\}$.

Definition 1.8. An even-symmetric (resp. odd-symmetric) $\mathbb{Z}_{2}$-graded quasialgebra $(A, B)$ is an $\mathbb{Z}_{2}$-graded quasialgebra $A$ provided with an even (resp. odd), supersymmetric, non-degenerate, and associative bilinear form $B$. The form $B$ is called an even-symmetric (resp. odd-symmetric) structure on $A$.

It is clear that an even-symmetric structure on $A$ is symmetric in even part and skew-symmetric in odd part, and also that an odd-symmetric structure on $A$ is symmetric.

Definition 1.9. Let $(A, B)$ be a homogeneous (even or odd)-symmetric antiassociative quasialgebra and $I$ a graded two-sided ideal of $A$.

(i) $I$ is non-degenerate if $\left.B\right|_{I \times I}$ is a non-degenerate bilinear form;

(ii) $(A, B)$ is $B$-irreducible if $A$ contains no non-degenerate graded two-sided ideal different of $\{0\}$ and $A$;

(iii) $I$ is $B$-irreducible if $I$ is non-degenerate and $I$ contains no non-degenerate graded two-sided ideal of $A$ different of $\{0\}$ and $I$.

Recall that $I \subseteq A$ is a graded two-sided ideal if $I=I_{\overline{0}} \oplus I_{\overline{1}}$, where $I_{\overline{0}}=I \cap A_{\overline{0}}$ and $I_{\overline{1}}=I \cap A_{\overline{1}}$. Hereafter in this section, most of the proofs of the results are adaptations of those for homogeneous symmetric associative superalgebras (see [5]), so are omitted.

Proposition 1.10. Every homogeneous (even or odd) symmetric antiassociative quasialgebra is an orthogonal direct sum of B-irreducible graded two-sided ideals. 
Due to the previous proposition, we may restrict our attention to $B$-irreducible homogeneous symmetric antiassociative quasialgebra.

Definition 1.11. An antiassociative quasialgebra $A$ is simple if $A^{2} \neq\{0\}$ and it contains no non-trivial graded two-sided ideals.

Definition 1.12. A graded two-sided ideal $I$ of an antiassociative quasialgebra $A$ is minimal if $I$ is not trivial and it contains no graded two-sided ideal of $A$ different of $\{0\}$.

The annihilator of a $\mathbb{Z}_{2}$-graded quasialgebra $A$ is the graded ideal of $A$ defined by

$$
\operatorname{Ann}(A):=\{x \in A: x y=y x=0, \forall y \in A\} .
$$

Lemma 1.13. If $A$ is a $\mathbb{Z}_{2}$-graded quasialgebra with identity then Ann $(A)=\{0\}$.

Proof. Take $x \in A n n(A)$. Denoting, as usual, by 1 the identity of $A$ we obtain that $x=x 1=0$ as required

Lemma 1.14. If I is a minimal graded two-sided ideal of a homogeneous symmetric antiassociative quasialgebra $A$ then $I$ is simple or $I$ is with null product such that $A I=I=I A$ or $I=\mathbb{K} x$, where $x$ is a homogeneous element of the annihilator $\operatorname{Ann}(A)$.

Lemma 1.15. Let $(A, B)$ be a non-simple B-irreducible homogeneous (even or odd) symmetric antiassociative quasialgebra such that $\operatorname{dim}(A)>1$. Let $I$ be a minimal graded two-sided ideal of $A$ and $J$ its orthogonal with respect to $B$. Then

(i) A/J is a simple antiassociative quasialgebra or a one-dimensional superalgebra with null product;

(ii) Furthermore, $A / J$ is a one-dimensional superalgebra with null product if and only if $I=\mathbb{K} x$, where $x$ is a homogeneous element of $\operatorname{Ann}(A)$.

We have a characterization of homogeneous (even or odd) symmetric antiassociative quasialgebras similar to the one obtained in the associative superalgebra case [4]. If $A=A_{\overline{0}} \oplus A_{\overline{1}}$ is an antiassociative quasialgebra, then $A_{\overline{0}}$ and $A_{\overline{1}}$ has a structure of $A_{\overline{0}}$-bimodules by means of $(L, R)$, where for $x \in A_{\overline{0}}$, we define $L(x):=L_{x}$ and $R(x):=R_{x}$. Moreover, $A_{\overline{0}}^{*}$ and $A_{\overline{1}}^{*}$ also have a structure of $A_{\overline{0}}$-bimodules by means of $\left(L^{*}, R^{*}\right)$ such that for $x \in A_{\overline{0}}$, we define $L^{*}(x)(f):=f \circ R_{x}$ and $R^{*}(x)(f):=f \circ L_{x}$, for all $f \in A_{i}^{*}$ and $i \in\{\overline{0}, \overline{1}\}$.

Proposition 1.16. Let $A$ be an antiassociative quasialgebra. $A$ is endowed with an even-symmetric structure if and only if there exist two isomorphisms of $A_{\overline{0}}$ bimodules $\phi_{\overline{0}}: A_{\overline{0}} \longrightarrow A_{\overline{0}}^{*}$ and $\phi_{\overline{1}}: A_{\overline{1}} \longrightarrow A_{\overline{1}}^{*}$ such that

$$
\begin{aligned}
& \phi_{i}(x)(y)=(-1)^{i} \phi_{i}(y)(x), \quad \forall x, y \in A_{i}, i \in\{\overline{0}, \overline{1}\}, \\
& \phi_{\overline{1}}(x y)(z)=\phi_{\overline{0}}(x)(y z), \quad \forall x \in A_{\overline{0}}, y, z \in A_{\overline{1}} .
\end{aligned}
$$

Proposition 1.17. Let $A$ be an antiassociative quasialgebra. $A$ is equipped with an odd-symmetric structure if and only if there exists an isomorphism of $A_{\overline{0}}$-bimodules $\phi: A_{\overline{1}} \longrightarrow A_{\overline{0}}^{*}$ such that

$$
\phi(x)(y z)=\phi(z)(x y), \quad \forall x, y, z \in A_{\overline{1}} .
$$

Proof. The linear map $\phi: A_{\overline{1}} \longrightarrow A_{\overline{0}}^{*}$ defined by $\phi(x)=B(x,),. \forall x \in A_{\overline{1}}$, is an isomorphism of $A_{\overline{0}}$-bimodules. 
The previous characterization of even (and odd) symmetric antiassociative quasialgebras presented in Proposition 1.16 (and Proposition 1.17, respectively) enables us to provide the next result of incompatibility of even-symmetric and odd-symmetric structures.

Proposition 1.18. Any antiassociative quasialgebra with non-null product does not admit simultaneously even-symmetric and odd-symmetric structures.

\section{Simple ANTIASsociative QUASIAlgebras With HOMOGENEOUS STRUCTURES}

First we inquire which of the simple antiassociative quasialgebras admit homogeneous structures. The following list contains all simple antiassociative quasialgebras (see [1] and [3]):

1) Take a division aaq-algebra $\Delta=\Delta_{\overline{0}} \oplus \Delta_{\overline{1}}(\simeq\langle D, \sigma, a\rangle)$ and a natural number $n$. Let $\operatorname{Mat}_{n}(\Delta)$ be the $\mathbb{Z}_{2}$-graded algebra of the $n \times n$ matrices over $\Delta$ with the natural $\mathbb{Z}_{2}$-gradation inherited from $\Delta$, whose even part is $\operatorname{Mat}_{n}\left(\Delta_{\overline{0}}\right)$ and the odd part $\operatorname{Mat}_{n}(\Delta)_{\overline{1}}$ is isomorphic to $\operatorname{Mat}_{n}\left(\Delta_{\overline{0}}\right) u$. Consider $\operatorname{Mat}_{n}(\Delta)=\operatorname{Mat}_{n}\left(\Delta_{\overline{0}}\right) \oplus$ $\operatorname{Mat}_{n}\left(\Delta_{\overline{0}}\right) u$ equipped with multiplication defined by

$$
\begin{aligned}
& A(B u)=(A B) u \\
& (A u) B=(A \bar{B}) u, \\
& (A u)(B u)=a A \bar{B}, \quad \forall A, B \in \operatorname{Mat}_{n}\left(\Delta_{\overline{0}}\right),
\end{aligned}
$$

where the matrix $\bar{B}$ is obtained from the matrix $B=\left[b_{i j}\right]_{1 \leq i, j \leq n}$ by replacing the term $b_{i j}$ by $\sigma\left(b_{i j}\right)$, for all $i, j \in\{1, \ldots, n\}$. Then $\operatorname{Mat}_{n}(\Delta)$ is a simple antiassociative quasialgebra.

2) Take a division algebra $D$ and two natural numbers $n$ and $m$. Consider the set of $(n+m) \times(n+m)$ matrices over $D$ denominated by $\widetilde{\operatorname{Mat}}_{n, m}(D)$ with $\mathbb{Z}_{2}$-gradation

$$
\begin{aligned}
\widetilde{\operatorname{Mat}}_{n, m}(D)_{\overline{0}} & =\left\{\left(\begin{array}{cc}
a & 0 \\
0 & b
\end{array}\right): a \in \operatorname{Mat}_{n}(D), b \in \operatorname{Mat}_{m}(D)\right\} \\
\widetilde{\operatorname{Mat}}_{n, m}(D)_{\overline{1}} & =\left\{\left(\begin{array}{cc}
0 & v \\
w & 0
\end{array}\right): v \in \operatorname{Mat}_{n \times m}(D), w \in \operatorname{Mat}_{m \times n}(D)\right\},
\end{aligned}
$$

and multiplication given by

$$
\left(\begin{array}{ll}
a_{1} & v_{1} \\
w_{1} & b_{1}
\end{array}\right)\left(\begin{array}{cc}
a_{2} & v_{2} \\
w_{2} & b_{2}
\end{array}\right)=\left(\begin{array}{cc}
a_{1} a_{2}+v_{1} w_{2} & a_{1} v_{2}+v_{1} b_{2} \\
w_{1} a_{2}+b_{1} w_{2} & -w_{1} v_{2}+b_{1} b_{2}
\end{array}\right)
$$

This multiplication is essentially the usual matrix multiplication apart from the minus sign. Then $\widetilde{\mathrm{Mat}}_{n, m}(D)$ is a simple antiassociative quasialgebra.

Proposition 2.1. The simple antiassociative quasialgebra $\operatorname{Mat}_{n}(\Delta)$ (with $n \geq 2$ ) does not admit an even-symmetric structure.

Proof. Consider the canonical basis $\left\{E_{i j}, 1 \leq i, j \leq n\right\}$ of $\operatorname{Mat}_{n}\left(\Delta_{\overline{0}}\right)$, where as usual $E_{i j}$ represents the matrix with the identity 1 in the $(i, j)$-position and zeros elsewhere. We note that $E_{i j} E_{l k}=\delta_{j l} E_{i k}$, where $\delta_{i j}$ is the Kronecker symbol. Let us assume that $\operatorname{Mat}_{n}(\Delta)$ admits an even-symmetric structure $B$. Since $B$ restrict to the odd part $\operatorname{Mat}_{n}\left(\Delta_{\overline{0}}\right) u$ is skew-symmetric we get that

$$
0=B\left(E_{11} u, E_{11} u\right)=B\left(1,\left(E_{11} u\right)\left(E_{11} u\right)\right)=B\left(1, a E_{11} \overline{E_{11}}\right)=a B\left(E_{11}, E_{11}\right),
$$

so $B\left(E_{11}, E_{11}\right)=0$. Now, following the same reasoning as in [5, Proposition 3.1], we show that $E_{11}$ is in the orthogonal of $\operatorname{Mat}_{n}(\Delta)$ with respect to $B$, which is a contradiction. 
Proposition 2.2. The simple antiassociative quasialgebra $\widetilde{M a t}_{n, m}(D)$, with $D$ a commutative division algebra, admits an even-symmetric structure.

Proof. Considering the usual trace form over the algebra of matrices without gradation, we define a bilinear form $B$ on the superalgebra $\widetilde{\mathrm{Mat}}_{n, m}(D)$ in the following way:

$B\left(M_{1}, M_{2}\right)=\operatorname{tr}\left(a_{1} a_{2}\right)+\operatorname{tr}\left(b_{1} b_{2}\right), \forall M_{1}=\left(\begin{array}{cc}a_{1} & 0 \\ 0 & b_{1}\end{array}\right), M_{2}=\left(\begin{array}{cc}a_{2} & 0 \\ 0 & b_{2}\end{array}\right) \in \widetilde{\operatorname{Mat}}_{n, m}(D)_{\overline{0}}$

$B\left(N_{1}, N_{2}\right)=\operatorname{tr}\left(c_{1} d_{2}\right)-\operatorname{tr}\left(d_{1} c_{2}\right), \forall N_{1}=\left(\begin{array}{cc}0 & c_{1} \\ d_{1} & 0\end{array}\right), N_{2}=\left(\begin{array}{cc}0 & c_{2} \\ d_{2} & 0\end{array}\right) \in \widetilde{\operatorname{Mat}}_{n, m}(D)_{\overline{1}}$

$B\left(\widetilde{\operatorname{Mat}}_{n, m}(D)_{\overline{0}}, \widetilde{\operatorname{Mat}}_{n, m}(D)_{\overline{1}}\right)=\{0\}$.

We easily show that $B$ is even super-symmetric and non-degenerate, as in [5] for associative case. Let us take care about the $B$-associativity. For all $M=\left(\begin{array}{cc}a & 0 \\ 0 & b\end{array}\right) \in$ $\widetilde{\operatorname{Mat}}_{n, m}(D)_{\overline{0}}$ and $N_{1}=\left(\begin{array}{cc}0 & c_{1} \\ d_{1} & 0\end{array}\right), N_{2}=\left(\begin{array}{cc}0 & c_{2} \\ d_{2} & 0\end{array}\right) \in \widetilde{\operatorname{Mat}}_{n, m}(D)_{\overline{1}}$ we have

$$
B\left(M N_{1}, N_{2}\right)=\operatorname{tr}\left(\begin{array}{cc}
\left(a c_{1}\right) d_{2} & 0 \\
0 & -\left(b d_{1}\right) c_{2}
\end{array}\right)=\operatorname{tr}\left(\left(a c_{1}\right) d_{2}\right)-\operatorname{tr}\left(\left(b d_{1}\right) c_{2}\right) .
$$

On the other hand, we infer that

$$
B\left(M, N_{1} N_{2}\right)=\operatorname{tr}\left(\begin{array}{cc}
a\left(c_{1} d_{2}\right) & 0 \\
0 & -b\left(d_{1} c_{2}\right)
\end{array}\right)=\operatorname{tr}\left(a\left(c_{1} d_{2}\right)\right)-\operatorname{tr}\left(b\left(d_{1} c_{2}\right)\right),
$$

which guarantees that $B$ is associative, completing the proof.

Remark 2.3. Due to the incompatibility proved on Proposition 1.18, we know that $\widetilde{\mathrm{Mat}}_{n, m}(D)$, with $D$ a commutative division algebra, does not admit an oddsymmetric structure.

\section{ODD-SYMMETRIC ANTIASSOCIATIVE QUASIALGEBRAS}

In this section we study odd-symmetric antiassociative quasialgebras in order to present a description of this class of antiassociative quasialgebras. Here it is important to emphasize that we are dealing with algebras over a field of characteristic different from 2 .

Proposition 3.1. If $A=A_{\overline{0}} \oplus A_{\overline{1}}$ is an odd-symmetric antiassociative quasialgebra over a field of characteristic not 2 then $A_{\overline{1}}^{2} A_{\overline{1}}=\{0\}$. Furthermore, any antiassociative quasialgebra admitting an odd-symmetric structure is an associative superalgebra.

Proof. Let $B$ an odd-symmetric structure on $A$. Using associativity of $B$ and antiassociativity of the algebra $A$, for $x, y, z \in A_{\overline{1}}$ and $t \in A_{\overline{0}}$ we have that

$$
B((x y) z, t)=-B(x(y z), t)=-B(x,(y z) t)=-B(x, y(z t))=-B((x y) z, t),
$$

hence $B((x y) z, t)=0$, because characteristic of the field is not 2 . Since $B$ is odd and non-degenerate then $(x y) z=0$, as required. Applying Proposition 1.4, it follows that $A$ is an associative superalgebra, completing the proof.

Corollary 3.2. If $A=A_{\overline{0}} \oplus A_{\overline{1}}$ is an odd-symmetric antiassociative quasialgebra over a field of characteristic not 2 then $A_{\overline{1}}^{2} \subseteq \operatorname{Ann}(A)$. 
Proof. Assume that $B$ is an odd-symmetric structure on $A$. Using associativity and supersymmetry of $B$ and antiassociativity of the algebra $A$, for $x, y, z \in A_{\overline{1}}$ and $t \in A_{\overline{0}}$ we get

$$
B((x y) t, z)=B(x(y t), z)=B(z, x(y t))=B(z x, y t)=B((z x) y, t)=0,
$$

because $A_{\overline{1}}^{2} A_{\overline{1}}=\{0\}$ by Proposition 3.1. Since $B$ is odd and $B\left(A_{\overline{1}}^{2} A_{\overline{0}}, A_{\overline{1}}\right)=0$, we have that $A_{\overline{1}}^{2} A_{\overline{0}}=\{0\}$. As $A_{\overline{1}}^{2} A_{\overline{0}}=\{0\}$ and $A_{\overline{1}}^{2} A_{\overline{1}}=\{0\}$ we conclude that $A_{\overline{1}}^{2} \subseteq \operatorname{Ann}(A)$ as desired.

Remark 3.3. The simple antiassociative quasialgebra $\operatorname{Mat}_{n}(\Delta)$ (with $n \geq 2$ ) does not admit an odd-symmetric structure because $A_{\overline{1}}^{2} A_{\overline{1}}$ does not reduce to zero. Indeed, for all $A, B, C \in \operatorname{Mat}_{n}\left(\Delta_{\overline{0}}\right)$ we have $((A u)(B u)) C u=(a A \bar{B}) C u=(a(A \bar{B}) C) u$ and $a(A \bar{B}) C$ is not necessarily zero.

Remark 3.4. We note that any odd-symmetric antiassociative quasialgebra is an associative superalgebra. However, not all odd-symmetric associative superalgebras are antiassociative quasialgebras. In fact, $Q:=Q_{n}(\mathbb{K})$ (with $n \geq 1$ ) is a simple odd-symmetric associative superalgebra (see [5]) such that $Q_{\overline{1}}^{2} Q_{\overline{1}}$ is not necessarily zero.

An odd-symmetric antiassociative quasialgebra $A=A_{\overline{0}} \oplus A_{\overline{1}}$ actually verifies one of the following two conditions:

(1) $A_{\overline{1}} \neq\{0\}$ and $A_{\overline{1}}^{2}=\{0\}$, so $A$ is a trivial antiassociative quasialgebra;

(2) $A_{1}^{2} \neq\{0\}$, the most relevant case.

Now we present an interesting example of a non trivial odd-symmetric antiassociative quasialgebra that is in the intersection of antiassociative quasialgebras with associative superalgebras, as we know from Proposition 3.1.

Example 3.5. Let $A=A_{\overline{0}} \oplus A_{\overline{1}}$ be a $\mathbb{Z}_{2}$-graded vector space such that $\operatorname{dim} A_{\overline{0}}=$ $\operatorname{dim} A_{\overline{1}}=n$. Let $\left\{e_{1}, e_{2}, \ldots, e_{n}\right\}$ be a basis of $A_{\overline{0}}$ and $\left\{v_{1}, v_{2}, \ldots, v_{n}\right\}$ be a basis of $A_{\overline{1}}$. We consider the symmetric bilinear map $A \times A \longrightarrow A$ defined by $e_{i} e_{j}=e_{i+j}$, if $i+j \leq n, e_{i} v_{j}=v_{j-i}$, if $i<j, v_{n}^{2}=e_{n}$, and all other products are zero. It is clear that the even part $A_{\overline{0}}$ is a nilpotent associative algebra. We prove that $A_{\overline{1}}$ is an associative $A_{\overline{0}}$-quasi-bimodule acting non trivially in $A_{\overline{0}}$. Let $e_{i}, e_{j}, v_{k}$ with $i+j<k$, then we have successively

$$
\begin{aligned}
& \left(e_{i} e_{j}\right) v_{k}=e_{i+j} v_{k}=v_{k-i-j}=e_{i} v_{k-j}=e_{i}\left(e_{j} v_{k}\right), \\
& \left(e_{i} v_{k}\right) e_{j}=v_{k-i} e_{j}=v_{k-i-j}=e_{i} v_{k-j}=e_{i}\left(v_{k} e_{j}\right), \\
& \left(v_{k} e_{i}\right) e_{j}=v_{k-i} e_{j}=v_{k-i-j}=v_{k} e_{i+j}=v_{k}\left(e_{i} e_{j}\right) .
\end{aligned}
$$

We can easy prove that $A$ with this multiplication is an antiassociative quasialgebra. We point out the following: as $A_{\overline{1}}^{2}=\left\langle e_{n}\right\rangle$ and $e_{n} \in A n n(A)$ (as $n+i>n$ we have $e_{n} e_{i}=0$, and since $n \geq i$ we get $\left.e_{n} v_{i}=0\right)$ we conclude that $A_{\overline{1}}^{2} A_{\overline{1}}=\{0\}$. Now consider $B: A \times A \longrightarrow \mathbb{K}$ the symmetric bilinear form given by $B\left(v_{i}, e_{j}\right)=\delta_{i j}$ for all $i, j \in\{1, \ldots, n\}$, where as usual $\delta_{i j}$ denotes the Kronecker symbol, and all other products are zero. With some calculations we can see that $B$ is an oddsymmetric structure on $A$ and consequently $(A, B)$ is a non trivial odd-symmetric antiassociative quasialgebra.

First we deal with odd-symmetric antiassociative quasialgebras $A=A_{\overline{0}} \oplus A_{\overline{1}}$ such that $A_{\overline{1}}^{2} \neq\{0\}$. Notice that applying Lemma 1.13 and Corollary 3.2, we know that $A$ does not have identity. Now we recall the notion of generalized double extension of an odd-symmetric associative superalgebra described by I. Ayadi and S. Benayadi (see Theorem 5.7 in [5]). 
Theorem 3.6. Let $\{W, B, V, \mu, \lambda, \gamma\}$ be a context of generalized double extension of the odd-symmetric associative superalgebra $(W, B)$ by the associative superalgebra $V$ (see the construction summarized in Definition 5.6 of [5]). Consider on the $\mathbb{Z}_{2}$-graded vector space $A:=V \oplus W \oplus V^{*}$ the product defined by

$$
\begin{aligned}
(v+x+f) \cdot\left(v^{\prime}+y+g\right) & =v \star v^{\prime}+\lambda\left(v, v^{\prime}\right)+\gamma\left(v, v^{\prime}\right)+x * y+\psi(x, y)+\mu(v)(y) \\
& +\nu(v, y)+(-1)^{\bar{x}} \bar{y} g R_{v}+\mu^{\prime}\left(v^{\prime}\right)(x)+\nu^{\prime}\left(x, v^{\prime}\right)+f \circ L_{v^{\prime}},
\end{aligned}
$$

and the supersymmetric bilinear form

$$
\widetilde{B}\left(v+x+f, v^{\prime}+y+g\right)=B(x, y)+g(v)+f\left(v^{\prime}\right)
$$

for all $v+x+f \in A_{\bar{x}}, v^{\prime}+y+g \in A_{\bar{y}}$. Then $(A, ., \widetilde{B})$ is an odd-symmetric associative superalgebra which is called the generalized double extension of $(W, B)$ by $V$ by means of $(\mu, \lambda, \gamma)$.

We use the notion of generalized double extension of an odd-symmetric associative superalgebra and again Corollary 3.2 to give the following result.

Proposition 3.7. Let $\left(A=A_{\overline{0}} \oplus A_{\overline{1}}, B\right)$ be a B-irreducible odd-symmetric antiassociative quasialgebra with $A_{\overline{1}}^{2} \neq\{0\}$. Then $A$ is a generalized double extension of an odd-symmetric antiassociative quasialgebra, by the one-dimensional superalgebra with null product.

Proof. Let $\left(A=A_{\overline{0}} \oplus A_{\overline{1}}, B\right)$ be a $B$-irreducible odd-symmetric antiassociative quasialgebra with $A_{\overline{1}}^{2} \neq\{0\}$. Applying Corollary 3.2, we know that $A n n(A) \neq\{0\}$. So $\left(A=A_{\overline{0}} \oplus A_{\overline{1}}, B\right)$ is a $B$-irreducible odd-symmetric associative superalgebra with $\operatorname{Ann}(A) \neq\{0\}$. Recalling the reasoning made in the associative case, we take an even non-zero element $e \in A_{\overline{1}}^{2} \subseteq \operatorname{Ann}(A)$, consider $I:=\mathbb{K} e$ and denote by $J$ the orthogonal of $I$ with respect to $B$. Since $B$ is an odd and non-degenerate bilinear form there exists an odd element $d$ such that $B(e, d) \neq 0$. Then $A$ is a generalized double extension $P\left(V^{*}\right) \oplus L \oplus V$ of an odd-symmetric associative superalgebra $L$ by the one-dimensional superalgebra $V:=\mathbb{K} d$ with null product. As $A$ is an odd-symmetric antiassociative quasialgebra, then $A_{\overline{1}}^{2} A_{\overline{1}}=\{0\}$ and so $L_{\overline{1}}^{2} L_{\overline{1}}=\{0\}$. Therefore, $L$ is an odd-symmetric associative superalgebra such that $L_{\overline{1}}^{2} L_{\overline{1}}=\{0\}$, consequently $L$ is an odd-symmetric antiassociative quasialgebra as required.

Corollary 3.8. If $\left(A=A_{\overline{0}} \oplus A_{\overline{1}}, B\right)$ is a $B$-irreducible odd-symmetric antiassociative quasialgebra such that $A_{\overline{1}}^{2} \neq\{0\}$, then it is obtained from an odd-symmetric antiassociative quasialgebra $L=L_{\overline{0}} \oplus L_{\overline{1}}$ such that $L_{\overline{1}}^{2}=\{0\}$ by a finite number of generalized double extension of an odd-symmetric associative superalgebra by the one-dimensional superalgebra with null product and/or orthogonal direct sums.

Secondly, we take care of the odd-symmetric antiassociative quasialgebras $A=$ $A_{\overline{0}} \oplus A_{\overline{1}}$ with $A_{\overline{1}}^{2}=\{0\}$.

Definition 3.9. Let $A$ be an associative algebra. We consider $P\left(A^{*}\right)$ the $\mathbb{Z}_{2}$-graded vector space with graduation

$$
\left(P\left(A^{*}\right)\right)_{\overline{0}}=\{0\},\left(P\left(A^{*}\right)\right)_{\overline{1}}=A^{*},
$$

where, as usual, $A^{*}$ denotes the dual space of $A$.

Proposition 3.10. Let $\left(A=A_{\overline{0}} \oplus A_{\overline{1}}, B\right)$ be an odd-symmetric antiassociative quasialgebra with $A_{\overline{1}}^{2}=\{0\}$. Then $A$ is semi-direct product of an associative algebra $\bar{A}$ by $P\left(\bar{A}^{*}\right)$ by means of $\left(L^{*}, R^{*}\right)$, meaning that, $A=\bar{A} \oplus P\left(\bar{A}^{*}\right)$ is odd-symmetric 
associative superalgebra with product and odd-symmetric structure $B$ on $A$ defined respectively by:

$$
\begin{aligned}
& (x+f)(y+h)=x y+L^{*}(x)(h)+R^{*}(y)(f) \\
& B(f, x)=B(x, f)=f(x), B(\bar{A}, \bar{A})=B\left(P\left(\bar{A}^{*}\right), P\left(\bar{A}^{*}\right)\right)=\{0\},
\end{aligned}
$$

for all $x, y \in \bar{A}$ and $f, h \in P\left(\bar{A}^{*}\right)$.

Proof. Since $\left(A=A_{\overline{0}} \oplus A_{\overline{1}}, B\right)$ is an odd-symmetric antiassociative quasialgebra, from Proposition 1.17 we have that $A_{\overline{1}}$ and $A_{\overline{0}}^{*}$ are isomorphic as $A_{\overline{0}}$-bimodules where the corresponding isomorphism $\phi: A_{\overline{1}} \longrightarrow A_{\overline{0}}^{*}$ is given by $\phi(x)=B(x,$.$) ,$ $\forall x \in A_{\overline{1}}$. Since $A_{\overline{1}}^{2}=\{0\}$, the multiplication in $A$ for $x, y \in A_{\overline{0}}$ and $a, b \in A_{\overline{1}}$ is

$$
(x+a)(y+b)=x y+x b+a y .
$$

Using the isomorphism between $A_{\overline{1}}$ and $A_{\overline{0}}^{*}$, the multiplication may be rewritten as

$$
(x+B(a, .))(y+B(b, .))=x y+B(x b, .)+B(a y, .) .
$$

For $z \in A_{\overline{0}}$, we obtain that

$$
\begin{aligned}
B(x b, z) & =B(x, b z)=B(b z, x)=B(b, z x)=B(b, .)(z x) \\
& =B(b, .) \circ R_{x}(z)=L^{*}(x)(B(b, .))(z)
\end{aligned}
$$

and similar

$$
B(a y, z)=B(a, y z)=B(a, .)(y z)=B(a, .) \circ L_{y}(z)=R^{*}(y)(B(a, .))(z) .
$$

Therefore

$$
(x+B(a, .))(y+B(b, .))=x y+L^{*}(x)(B(b, .))+R^{*}(y)(B(a, .)) .
$$

Furthermore, from the isomorphism between $A_{\overline{1}}$ and $A_{\overline{0}}^{*}$, the odd-symmetric structure on $A$ may be interpreted as $B(a, x)=B(a,).(x)$ where $B(a,$.$) belongs to A_{\overline{0}}^{*}$, for $x \in A_{\overline{0}}$ and $a \in A_{\overline{1}}$. Thus $A$ is the semi-direct product of the associative algebra $A_{\overline{0}}$ by $P\left(A_{\overline{0}}^{*}\right)$ by means of $\left(L^{*}, R^{*}\right)$ as required.

Now we are in position to establish the main result concerning odd-symmetric antiassociative quasialgebras.

Corollary 3.11. Let $\left(A=A_{\overline{0}} \oplus A_{\overline{1}}, B\right)$ be an odd-symmetric antiassociative quasialgebra. Then $A$ is either obtained by a finite sequence of generalized double extension of an odd-symmetric associative superalgebra by one-dimensional superalgebra with null product and/or by semi-direct product of an associative algebra $\bar{A}$ by $P\left(\bar{A}^{*}\right)$ by means of $\left(L^{*}, R^{*}\right)$ and/or by orthogonal direct sums.

The odd-symmetric associative superalgebras were studied in [5]. In particular, the authors presented an interesting description of odd-symmetric associative superalgebras whose even part is a semi-simple bimodule, without the use of the notions of double extensions. As any $\mathbb{Z}_{2}$-graded quasialgebra is either an associative superalgebra or an antiassociative quasialgebra, we conclude that the class of odd-symmetric $\mathbb{Z}_{2}$-graded quasialgebras have been completely covered.

\section{Generalized double extension of even-Symmetric antiassociative QUASIALGEBRAS}

The generalized double extension is performed in two distinct steps, a central extension followed by a generalized semidirect product. This extension will help us to present a description of even-symmetric antiassociative quasialgebras.

First we explain the notion of central extension of an antiassociative quasialgebra. Let $(W, *)$ be an antiassociative quasialgebra, $V$ a $\mathbb{Z}_{2}$-graded vector space, and $h: W \times W \longrightarrow V$ an even bilinear map. Consider the $\mathbb{Z}_{2}$-graded vector space 
$W \oplus V$ (with the grading $(W \oplus V)_{\overline{0}}=W_{\overline{0}} \oplus V_{\overline{0}}$ and $\left.(W \oplus V)_{\overline{1}}=W_{\overline{1}} \oplus V_{\overline{1}}\right)$ provided with the product defined by

$$
(x+v) \times\left(y+v^{\prime}\right)=x * y+h(x, y), \quad \forall x+v \in(W \oplus V)_{\bar{x}}, y+v^{\prime} \in(W \oplus V)_{\bar{y}} .
$$

Then $(W \oplus V, \times)$ is an antiassociative quasialgebra if and only if $h(x * y, z)=$ $(-1)^{\bar{x} \bar{y} \bar{z}} h(x, y * z)$, holds for all homogeneous elements $x, y, z$ in $W$. We note that $V$ is in $\operatorname{Ann}(W \oplus V)$, consequently in the center of $W \oplus V$. The antiassociative quasialgebra $(W \oplus V, \times)$ will be called central extension of $W$ by $V$ by means of $h$.

Now we introduce the tool of generalized semidirect product of an antiassociative quasialgebra by an other antiassociative quasialgebra. Let $(W, *)$ and $(V, \star)$ be two antiassociative quasialgebras. Consider $\eta: V \longrightarrow \operatorname{End}(W), \eta^{\prime}: V \longrightarrow \operatorname{End}(W)$ two even linear maps and $\epsilon: V \times V \longrightarrow W$ an even bilinear map verifying the following conditions:

$$
\begin{aligned}
& \left(p_{1}\right) \eta^{\prime}(v)(x * y)=(-1)^{\bar{x} \bar{y} \bar{v}} x * \eta^{\prime}(v)(y), \\
& \left(p_{2}\right) \eta^{\prime}(v)(x) * y=(-1)^{\bar{x} \bar{y}} x * \eta(v)(y), \\
& \left(p_{3}\right) \eta(v)(x) * y=(-1)^{\bar{x} \bar{y}} \eta(v)(x * y), \\
& \left(p_{4}\right)\left(\eta^{\prime}\left(v^{\prime}\right) \circ \eta(v)\right)(x)=(-1)^{\bar{x} \bar{v} \bar{v}^{\prime}}\left(\eta(v) \circ \eta^{\prime}\left(v^{\prime}\right)\right)(x), \\
& \left(p_{5}\right)\left(\eta^{\prime}\left(v^{\prime}\right) \circ \eta^{\prime}(v)\right)(x)=(-1)^{\bar{x} \bar{v} \bar{v}^{\prime}}\left(x * \epsilon\left(v, v^{\prime}\right)+\eta^{\prime}\left(v \star v^{\prime}\right)(x)\right), \\
& \left(p_{6}\right)\left(\eta(v) \circ \eta\left(v^{\prime}\right)\right)(x)=(-1)^{\bar{x} \bar{v} \bar{v}^{\prime}}\left(\epsilon\left(v, v^{\prime}\right) * x+\eta\left(v \star v^{\prime}\right)(x)\right), \\
& \left(p_{7}\right) \eta^{\prime}\left(v^{\prime \prime}\right)\left(\epsilon\left(v, v^{\prime}\right)\right)+\epsilon\left(v \star v^{\prime}, v^{\prime \prime}\right)=(-1)^{\bar{v} \bar{v}^{\prime} \bar{v}^{\prime \prime}}\left(\eta(v)\left(\epsilon\left(v^{\prime}, v^{\prime \prime}\right)\right)+\epsilon\left(v, v^{\prime} \star v^{\prime \prime}\right)\right),
\end{aligned}
$$

where $x, y$ are homogeneous elements of $W$ and $v, v^{\prime}, v^{\prime \prime}$ homogeneous elements of $V$. Consider on the $\mathbb{Z}_{2}$-graded vector space $W \oplus V$ the product defined in the following way:

$$
(x+v) \cdot\left(y+v^{\prime}\right)=v \star v^{\prime}+x * y+\eta(v)(y)+\eta^{\prime}\left(v^{\prime}\right)(x)+\epsilon\left(v, v^{\prime}\right),
$$

for all $x+v \in(W \oplus V)_{\bar{x}}, y+v^{\prime} \in(W \oplus V)_{\bar{y}}$. From conditions $\left(p_{1}\right)$ to $\left(p_{7}\right)$, it follows that $(W \oplus V,$.$) is an antiassociative quasialgebra.$

Definition 4.1. Let $W, V$ be two antiassociative quasialgebras and $\left(\eta, \eta^{\prime}, \epsilon\right)$ defined as above satisfying conditions $\left(p_{1}\right)$ to $\left(p_{7}\right)$. The triple $\left(\eta, \eta^{\prime}, \epsilon\right)$ will be called an admissible triple and the antiassociative quasialgebra $(W \oplus V,$.$) will be termed the$ generalized semidirect product of $W$ by $V$ by means of $\left(\eta, \eta^{\prime}, \epsilon\right)$.

Finally, we are prepared to present the concept of generalized double extension of an even-symmetric antiassociative quasialgebras. Consider $(W, *, B)$ an evensymmetric antiassociative quasialgebra and $(V, \star)$ an antiassociative quasialgebra. Let $\mu: V \longrightarrow \operatorname{End}(W)$ be an even linear map and $\lambda: V \times V \longrightarrow W$ an even bilinear map obeying to the following conditions:

$$
\begin{aligned}
& \mu^{\prime}(v)(x * y)=(-1)^{\bar{x} \bar{y} \bar{v}} x * \mu^{\prime}(v)(y), \\
& \left(\mu^{\prime}\left(v^{\prime}\right) \circ \mu(v)\right)(x)=(-1)^{\bar{x} \bar{v} \bar{v}^{\prime}}\left(\mu(v) \circ \mu^{\prime}\left(v^{\prime}\right)\right)(x), \\
& \mu^{\prime}\left(v \star v^{\prime}\right)(x)+x * \lambda\left(v, v^{\prime}\right)=(-1)^{\bar{x} \bar{v} \bar{v}^{\prime}}\left(\mu^{\prime}\left(v^{\prime}\right) \circ \mu^{\prime}(v)\right)(x), \\
& \mu^{\prime}\left(v^{\prime \prime}\right)\left(\lambda\left(v, v^{\prime}\right)\right)+\lambda\left(v \star v^{\prime}, v^{\prime \prime}\right)=(-1)^{\bar{v} \bar{v}^{\prime} \bar{v}^{\prime \prime}} \mu(v)\left(\lambda\left(v^{\prime}, v^{\prime \prime}\right)\right) \\
& +(-1)^{\bar{v} \bar{v}^{\prime} \bar{v}^{\prime \prime} \lambda\left(v, v^{\prime} \star v^{\prime \prime}\right),}
\end{aligned}
$$

whenever $x, y$ are homogeneous elements of $W, v, v^{\prime}, v^{\prime \prime}$ homogeneous elements of $V$, and $\mu^{\prime}: V \longrightarrow \operatorname{End}(W)$ is an even linear map defined by the relation

$$
B(\mu(v)(x), y)=(-1)^{\bar{v}(\bar{x}+\bar{y})} B\left(x, \mu^{\prime}(v)(y)\right), \quad \forall x \in W_{\bar{x}}, y \in W_{\bar{y}}, v \in V_{\bar{v}},
$$


meaning that, for all $v \in V_{\bar{v}}, \mu^{\prime}(v)$ is the adjoint map of $\mu(v)$ with respect to $B$. Let $\nu: V \times W \longrightarrow V^{*}$ and $\nu^{\prime}: W \times V \longrightarrow V^{*}$ be two even bilinear maps defined respectively by

$$
\nu(v, x)\left(v^{\prime}\right)=(-1)^{\bar{v}\left(\bar{x}+\bar{v}^{\prime}\right)} B\left(x, \lambda\left(v^{\prime}, v\right)\right) \quad \text { and } \quad \nu^{\prime}(x, v)\left(v^{\prime}\right)=B\left(x, \lambda\left(v, v^{\prime}\right)\right),
$$

where $x$ is an homogeneous element of $W$ and $v, v^{\prime}$ homogeneous elements of $V$. Consider the representation of $V$ on its dual $V^{*}$ by $f . v=f \circ L_{v}$ and $v \cdot f=(-1)^{\bar{v} \bar{f}} f \circ$ $R_{v}$, for all $v \in V_{\bar{v}}, f \in V^{*}{ }_{\bar{f}}$, and the bilinear map $\gamma: V \times V \longrightarrow V^{*}$ satisfying the two following conditions:

$\gamma\left(v, v^{\prime}\right)\left(v^{\prime \prime}\right)=(-1)^{\bar{v}\left(\bar{v}^{\prime}+\bar{v}^{\prime \prime}\right)} \gamma\left(v^{\prime}, v^{\prime \prime}\right)(v)$,

$B\left(\lambda\left(v, v^{\prime}\right), \lambda\left(v^{\prime}, v^{\prime \prime \prime}\right)\right)+\gamma\left(v \star v^{\prime}, v^{\prime \prime}\right)\left(v^{\prime \prime \prime}\right)+\gamma\left(v, v^{\prime}\right)\left(v^{\prime \prime} \star v^{\prime \prime \prime}\right)=$

$=(-1)^{\bar{v}} \overline{v^{\prime}} \overline{v^{\prime \prime}}+\bar{v}\left(\overline{v^{\prime}}+\bar{v}^{\bar{\prime}}+v^{\overline{\prime \prime \prime}}\right) B\left(\lambda\left(v^{\prime}, v^{\prime \prime}\right), \lambda\left(v^{\prime \prime \prime}, v\right)\right)+(-1)^{\bar{v} \bar{v}^{\prime} v^{\prime \prime}} \gamma\left(v, v^{\prime} \star v^{\prime \prime}\right)\left(v^{\prime \prime \prime}\right)$

$+(-1)^{\bar{v} \bar{v}^{\prime} \bar{v}^{\prime \prime}+\bar{v}\left(\overline{v^{\prime}}+\bar{v}^{\prime \prime}+v^{\overline{\prime \prime} \prime}\right)} \gamma\left(v^{\prime}, v^{\prime \prime}\right)\left(v^{\prime \prime \prime} \star v\right)$,

for homogeneous elements $v, v^{\prime}, v^{\prime \prime}, v^{\prime \prime \prime}$ of $V$. Let $\phi: W \times W \longrightarrow V^{*}$ be the even bilinear map defined by

$$
\phi(x, y)(v)=B\left(x, \mu^{\prime}(v)(y)\right), \quad \forall x, y \in W, v \in V .
$$

We summarize all the details above in the following definition:

Definition 4.2. Let $(W, B)$ be an even-symmetric antiassociative quasialgebra, $V$ an antiassociative quasialgebra and $(\mu, \lambda, \gamma)$ defined as above satisfying conditions (4.1) to (4.6). Then $\{W, B, V, \mu, \lambda, \gamma\}$ will be called a context of generalized double extension of the even-symmetric antiassociative quasialgebra $(W, B)$ by the antiassociative quasialgebra $V$.

Theorem 4.3. Let $\{W, B, V, \mu, \lambda, \gamma\}$ be a context of generalized double extension of the even-symmetric antiassociative quasialgebra $W$ by the antiassociative quasialgebra $V$. Consider on the $\mathbb{Z}_{2}$-graded vector space $A:=V \oplus W \oplus V^{*}$ the product defined by

$$
\begin{aligned}
(v+x+f) \cdot\left(v^{\prime}+y+g\right) & =v \star v^{\prime}+\lambda\left(v, v^{\prime}\right)+\gamma\left(v, v^{\prime}\right)+x * y+\phi(x, y)+\mu(v)(y) \\
& +\nu(v, y)+(-1)^{\bar{x}} \bar{y} \circ R_{v}+\mu^{\prime}\left(v^{\prime}\right)(x)+\nu^{\prime}\left(x, v^{\prime}\right)+f \circ L_{v^{\prime}},
\end{aligned}
$$

and the supersymmetric bilinear form

$$
\widetilde{B}\left(v+x+f, v^{\prime}+y+g\right)=B(x, y)+(-1)^{\bar{x}} \bar{y} g(v)+f\left(v^{\prime}\right),
$$

for all $v+x+f \in A_{\bar{x}}, v^{\prime}+y+g \in A_{\bar{y}}$. Then $(A, ., \widetilde{B})$ is an even-symmetric antiassociative quasialgebra such that the subspace $V^{*}$ is a totally isotropic graded ideal of $A$ and $W \oplus V^{*}$ its orthogonal with respect to $\widetilde{B}$. The even-symmetric antiassociative quasialgebra $(A, \widetilde{B})$ is called the generalized double extension of the even-symmetric antiassociative quasialgebra $(W, B)$ by the antiassociative quasialgebra $V$ by means of $(\mu, \lambda, \gamma)$.

Proof. Using (4.1) we show that $\phi(x * y, z)=(-1)^{\bar{x} \bar{y} \bar{z}} \phi(x, y * z)$, for homogeneous elements $x, y, z$ in $W$, thus $W \oplus V^{*}$ is an antiassociative quasialgebra which is the central extension of $W$ by $V^{*}$ by means of $\phi$. We define the even linear maps $\eta: V \longrightarrow \operatorname{End}\left(W \oplus V^{*}\right), \eta^{\prime}: V \longrightarrow \operatorname{End}\left(W \oplus V^{*}\right)$ and the even bilinear map $\epsilon: V \times V \longrightarrow W \oplus V^{*}$ in the following way:

$$
\begin{aligned}
& \eta(v)(x+f)=\mu(v)(x)+\nu(v, x)+(-1)^{\bar{x} \bar{v}} f \circ R_{v}, \\
& \eta^{\prime}(v)(x+f)=\mu^{\prime}(v)(x)+\nu^{\prime}(x, v)+f \circ L_{v}, \\
& \epsilon\left(v, v^{\prime}\right)=\lambda\left(v, v^{\prime}\right)+\gamma\left(v, v^{\prime}\right),
\end{aligned}
$$


whenever $x+f \in\left(W \oplus V^{*}\right)_{\bar{x}}, v \in V_{\bar{v}}, v^{\prime} \in V$. Using assertions (4.1) to (4.4) we show that $\left(\eta, \eta^{\prime}, \epsilon\right)$ is an admissible triple and therefore $A$ is the generalized semidirect product of $W \oplus V^{*}$ by $V$ by means of the triple $\left(\eta, \eta^{\prime}, \epsilon\right)$.

Let us assume in particular that $V:=\mathbb{K} e$ is a one-dimensional superalgebra with $e \star e=\alpha_{0} e$. Then $\{W, B, V, \mu, \lambda, \gamma\}$ a context of generalized double extension of a even-symmetric antiassociative quasialgebra $(W, B)$ by $\mathbb{K} e$ is defined in a simple way by $\left\{W, B, \mathbb{K} e, D, x_{0}, \gamma_{0}\right\}$, taking $D=\mu(e) \in \operatorname{End}(W)_{\bar{e}}, x_{0}=\lambda(e, e) \in W_{\overline{0}}$ and $\gamma_{0}=\gamma(e, e)(e) \in \mathbb{K}_{\bar{e}}$ (where $\mathbb{K}_{\overline{0}}=\mathbb{K}, \mathbb{K}_{\overline{1}}=\{0\}$ if $e$ is even, and $\mathbb{K}_{\overline{0}}=\{0\}, \mathbb{K}_{\overline{1}}=\mathbb{K}$ if $e$ is odd).

Corollary 4.4. Let $\left\{W, B, \mathbb{K} e, D, x_{0}, \gamma_{0}\right\}$ be a context of generalized double extension of an even-symmetric antiassociative quasialgebra $(W, B)$ by $\mathbb{K} e$. Consider the $\mathbb{Z}_{2}$-graded vector space $A:=\mathbb{K} e \oplus W \oplus \mathbb{K} e^{*}$ with the product defined as in Theorem 4.3 and the supersymmetric bilinear form $\widetilde{B}$ defined by $\left.\widetilde{B}\right|_{W \times W}=B, \widetilde{B}\left(e^{*}, e\right)=1$ and $\widetilde{B}(e, W)=\widetilde{B}\left(e^{*}, W\right)=0$. Then $(A, ., \widetilde{B})$ is an even-symmetric antiassociative quasialgebra and it is called the even (resp. odd) generalized double extension of $W$ by $\mathbb{K} e$ by means of $\left(D, x_{0}, \gamma_{0}\right)$ (resp. $\left(D, x_{0}\right)$ ) if $e$ is an even (resp. odd) homogeneous element in $A$.

\section{EVEn-SYMmETRIC ANTIASSOCIATIVE QUASIALGEBRAS WITH EVEN PART SEMI-SIMPLE BIMODULE}

Our goal is to present an inductive description of antiassociative quasialgebras admitting an even-symmetric structure and whose even part is a semi-simple bimodule . First, we give a characterization of minimal graded two-sided ideals of these algebras. Some of the proofs are similar to those in associative superalgebras case presented in [5], so we omit them.

Proposition 5.1. Let $\left(A=A_{\overline{0}} \oplus A_{\overline{1}}, B\right)$ be a non simple B-irreducible evensymmetric antiassociative quasialgebra such that $A_{\overline{0}}$ is semi-simple $A_{\overline{0}}$-bimodule. Suppose that $A$ is different from the one-dimensional algebra with null product. Then $I$ is a minimal graded two-sided ideal of $A$ if and only if $I=\mathbb{K} x$, where $x$ is a homogeneous element of $\operatorname{Ann}(A)$.

Proposition 5.2. Let $\left(A=A_{\overline{0}} \oplus A_{\overline{1}}, B\right)$ be an even-symmetric antiassociative quasialgebra such that $A_{\overline{0}}$ is semi-simple $A_{\overline{0}}$-bimodule. If $\operatorname{Ann}(A)=\{0\}$ then $A$ is orthogonal direct sums of simple even-symmetric antiassociative quasialgebras.

From the previous result, we realize that we have to study these antiassociative quasialgebras with $\operatorname{Ann}(A) \neq\{0\}$.

Lemma 5.3. Let $\left(A=A_{\overline{0}} \oplus A_{\overline{1}}, B\right)$ be an $B$-irreducible even-symmetric antiassociative quasialgebra such that $A_{\overline{0}}$ is semi-simple $A_{\overline{0}}$-bimodule, which is different of the one-dimensional algebra with null product. If $\operatorname{Ann}(A) \cap A_{\overline{0}} \neq\{0\}$ then $\operatorname{Ann}(A) \cap A_{\overline{0}}$ is enclosed strictly in $\operatorname{Ann}\left(A_{\overline{0}}\right)$ (meaning that $\operatorname{Ann}(A) \cap A_{\overline{0}} \varsubsetneqq \operatorname{Ann}\left(A_{\overline{0}}\right)$ ).

Proposition 5.4. Let $\left(A=A_{\overline{0}} \oplus A_{\overline{1}}, B\right)$ be a $B$-irreducible even-symmetric antiassociative quasialgebra such that $A_{\overline{0}}$ is semi-simple $A_{\overline{0}}$-bimodule, which is different of the one-dimensional algebra with null product. If $A n n(A) \cap A_{\overline{0}} \neq\{0\}$ (respectively $\left.\operatorname{Ann}(A) \cap A_{\overline{1}} \neq\{0\}\right)$ then $A$ is an elementary even generalized double extension (respectively odd generalized double extension) of an even-symmetric antiassociative quasialgebra $W$, with $W_{\overline{0}}$ semi-simple $W_{\overline{0}}$-bimodule, by the one-dimensional algebra with null product (respectively one-dimensional superalgebra with null even part). 
Now we state the inductive description of even-symmetric antiassociative quasialgebras with even part semi-simple bimodule. Consider the set $\mathfrak{U}$ formed by $\{0\}$, one-dimensional algebra with null product and $\widetilde{\mathrm{Mat}}_{n, m}(D)$.

Corollary 5.5. Let $\left(A=A_{\overline{0}} \oplus A_{\overline{1}}, B\right)$ be an even-symmetric antiassociatice quasialgebra with even part semi-simple $A_{\overline{0}}$-bimodule. Then $A$ is either an element of $\mathfrak{U}$ or $A$ is obtained from a finite number of elements of $\mathfrak{U}$ by a finite sequence of elementary even double extensions by the one-dimensional algebra with null product and/or odd generalized double extensions by the one-dimensional superalgebra with null even part and/or orthogonal direct sums.

\section{Even-SYMmETRIC ANTIASSOCIATIVE QUASIALGEBRAS}

This section is devoted to even-symmetric antiassociative quasialgebras in general. Our main purpose is to provide a description of this class of algebras.

Theorem 6.1. Let $(A, B)$ be a non-simple B-irreducible even-symmetric antiassociative quasialgebra such that $\operatorname{dim}(A)>1$. Let $I$ be a totally isotropic graded two-sided ideal of $A$ and $J$ its orthogonal with respect to $B$.

(i) Then J/I provided with natural multiplication and bilinear form given by

$$
\widetilde{B}(x+I, y+I)=B(x, y), \quad \forall x, y \in J,
$$

is an even-symmetric antiassociative quasialgebra.

(ii) If $A=J \oplus V$, where $V$ is a $\mathbb{Z}_{2}$-graded subspace of $A$, then $A$ is isomorphic to the generalized double extension of $(J / I, \widetilde{B})$ by $V$.

Proposition 6.2. Let $(A, B)$ be an non-simple B-irreducible even-symmetric antiassociative quasialgebra such that $\operatorname{dim}(A)>1$ such that $\operatorname{Ann}(A) \neq\{0\}$. Then $A$ is an even or odd generalized double extension of an even-symmetric antiassociative quasialgebra by one-dimensional superalgebra.

The proof of Theorem 6.1 and Proposition 6.2 are similar to those in associative superalgebras case (see [5]) so are omitted here.

Proposition 6.3. Let $(A, B)$ be an non-simple B-irreducible even-symmetric antiassociative quasialgebra such that $\operatorname{dim}(A)>1$. Then $A$ is a generalized double extension of an even-symmetric antiassociative quasialgebra by an element of $\left\{\operatorname{Mat}_{n}(\Delta), \widetilde{M a t}_{n, m}(D)\right\}$ (the set of simple antiassociative quasialgebras), or an even generalized double extension of an even-symmetric antiassociative quasialgebra by the one-dimensional algebra with null product and/or an odd generalized double extension of an even-symmetric antiassociative quasialgebra by the one-dimensional superalgebra with null even part.

Now the inductive description of even-symmetric antiassociative quasialgebras is an immediate consequence of the previous proposition. Let $\mathfrak{U}$ be the set formed by $\{0\}$, one-dimensional algebra with null product and $\widetilde{\mathrm{Mat}}_{n, m}(D)$.

Corollary 6.4. Any even-symmetric antiassociative quasialgebra $(A, B)$ is either an element of $\mathfrak{U}$ or obtained from finite number of elements of $\mathfrak{U}$ by finite sequence of generalized double extensions by elements of $\left\{\operatorname{Mat}_{n}(\Delta), \widetilde{M a t}_{n, m}(D)\right\}$ and/or even generalized double extension by the one-dimensional algebra with null product and/or odd generalized double extension by the one-dimensional superalgebra with null even part and/or orthogonal direct sums. 


\section{REFERENCES}

[1] H. Albuquerque, A. Elduque, and J.M. Pérez-Izquierdo, $\mathbb{Z}_{2}$-quasialgebras, Comm. Algebra 30 (2002), 2161-2174.

[2] H. Albuquerque and Shahn Majid, Quasialgebra structure of the octonions, J. Algebra 220 (1999), 188-224.

[3] H. Albuquerque and A. P. Santana, Simple quasiassociative algebras. Mathematical papers in honour of Eduardo Marques de Sá, Textos Mat. Sér. B, Univ. Coimbra, Coimbra 39 (2006), $135-145$.

[4] I. Ayadi, "Super-algèbres non Associatives avec des Structures Homogènes", Doctoral Thesis, Université Paul Verlaine-Metz, 2011.

[5] I. Ayadi and S. Benayadi, Associative superalgebras with homogeneous symmetric structures, Comm. Algebra 40 (2012), no. 4, 1234-1259.

[6] I. Ayadi and S. Benayadi, Symmetric Novikov superalgebras, J. Math. Phys. 51 (2010), no. 2, 023501, 15 pp.

[7] M.L. Racine, Primitive superalgebras with superinvolution, J. Algebra 206 (1998), 588-614.

[8] C.T.C. Wall, Graded Brauer groups, J. Reine Angew. Math. 213 (1964), 187-199.

Helena Albuquerque, CMuC, Departamento de Matemática, Universidade de Coimbra, Apartado 3008, 3001-454 Coimbra, Portugal E-mail address: lena@mat.uc.pt

Elisabete Barreiro, CMUC, Departamento de Matemática, Universidade de Coimbra, Apartado 3008, 3001-454 Coimbra, Portugal E-mail address: mefb@mat.uc. pt

Saïd Benayadi, Laboratoire de Mathmatiques et Applications de Metz, CNRS-UMR 7122, Université de Lorraine, Ile du Saulcy, 57045 Metz Cedex 1, FRANCE E-mail address: BENAYADI@UNIV-METZ.FR 